\title{
Solving the four index fully fuzzy transportation problem
}

\author{
Manal Hedid ${ }^{1, *}$ and Rachid Zitouni ${ }^{1}$ \\ ${ }^{1}$ Laboratory of Fundamental and Numerical Mathematics \\ Faculty of Sciences, University Ferhat Abbas Setif1, Setif 19000, Algeria \\ E-mail: 〈\{manal.hedid,rzitouni\}@univ-setif.dz〉
}

\begin{abstract}
In this paper, we will solve the four index fully fuzzy transportation problem $\left(F F T P_{4}\right)$ with some adapted classical methods. All problem's data will be presented as fuzzy numbers. In order to defuzificate these data, we will use the ranking function procedure. Our method to solve the FTPP $_{4}$ composed of two phases; in the first one, we will use an adaptation of well-known algorithms to find an initial feasible solution, which are the least cost, Russell's approximation and Vogel's approximation methods. In the second phase, we will test the optimality of the initial solution, if it is not optimal, we will improve it. A numerical analysis of the proposed methods is performed by solving different examples of different sizes; it is determined that they are stable, robust, and efficient. A proper comparative study between the adapted methods identifies the suitable method for solving $F F T P_{4}$.
\end{abstract}

Keywords: fuzzy arithmetic, fuzzy transportation problem, linear programming, multi-index problem

Received: June 26, 2020; accepted: October 19, 2020; available online: December 18, 2020

DOI: 10.17535 /crorr.2020.0016

\section{Introduction}

In the current dynamic market, companies must find the most efficient means of sending products to customers in the quantities requested as soon as possible at the lowest cost; this is the well-known transportation problem $(T P)$. The classical transportation problem has been widely studied [20] and then generalized to more than two indexes [18, 26, 27]. Based on its typology [18], TP can be classified into four groups: 2-index, 3 -index, 4 -index, and $n$-index. Previously, many efficient algorithms have been developed to identify the optimal total cost when the demands, offers, and unitary costs are known.

In real-world applications, there are many different situations where classical methods for solving $T P$ are no longer suitable because of the uncertainties of one or more parameters that cannot be accurately determined; this occurs owing to the imprecise tool of measure, lost and missing data, and even calculation errors. To overcome this problem, problem's data are presented with fuzzy numbers, for the purpose of modeling the uncertainties within the data, thus fuzzy mathematics becomes mandatory in solving this type of problems.

The theoretical foundations of fuzzy logic were established in the early 1965 by L- Zadeh [24]. Fuzzy logic is based on the association of the notion of "fuzzy subset" and "theory of possibilities". The world of reasoning in fuzzy logic is more intuitive than in binary logic; it allows designers to better understand natural phenomena and to model them using the definition of rules and membership functions in sets called "fuzzy sets". A fuzzy transportation problem $(F T P)$ is a problem where its data (e.g., costs and or offers and requests) are fuzzy quantities. In 1970, Bellman and Zadeh [2] first introduced the concept of decision-making in a fuzzy

${ }^{*}$ Corresponding author. 
environment. Eight years later, Zimmermann [25] showed that fuzzy linear programming algorithms were effective. In 1984, Chanas, Kolodziejkzy, and Machaj [3] presented a fuzzy linear programming model to solve the fuzzy transportation problem. Chanas and Kuchta (1996)[4] introduced a new concept for solving a transportation problem with fuzzy numbers data.

In 2004, Liu and Kao [11] described a method for solving FTP using the extension principle. Nagoor Gani and Abdul Razak (2006) [16] obtained a fuzzy solution for the two-stage cost minimizing of FTP in which availabilities and demands are trapezoidal fuzzy numbers. Pandian and Natarajan (2010) [17] proposed a fuzzy zero point method for finding an optimal solution for FTP, where all parameters are trapezoidal fuzzy numbers. Kumar and Kauar (2011) [7] proposed a new method for solving FTP by assuming that a decision maker was uncertain about the exact values of the transportation cost, availability, and demand of the product. Kumar and Murugesan (2012) [9] provided an optimal solution for FTP using triangular fuzzy numbers using a modified revised simplex method. In [10], P. Kumari performed a comparative study on the optimality of the initial solutions obtained by different available methods for the two-index fuzzy transportation problem. In 2020 [19], Jayanta Pratihar et al. proposed an algorithm that was based on Vogel's approximation method to solve the fuzzy transportation problem.

The two-index fuzzy transportation problem has been rigorously studied using fuzzy triangular numbers in $[1,9,15]$, intuitionistic fuzzy numbers in $[5,12,13,23]$, and fuzzy trapezoidal numbers in $[7,10,14,16,17,21,22]$. In addition, in 2019, Kumar et al. [8] used Pythagorean fuzzy numbers. However the efficiency of these algorithms was not tested for the multi-index fuzzy transportation problem. Authors in [6] proposed an adaptation of R. Zitouni's algorithm [27] for solving the four index fuzzy transportation problem.

In this paper, we will present three adapted algorithms to solve the four index fully fuzzy transportation problem $\left(\mathrm{FFTP}_{4}\right)$ using both trapezoidal and triangular fuzzy numbers. Using the ranking function of fuzzy availabilities, fuzzy requests, fuzzy means of transportation, fuzzy qualities of the goods, and fuzzy costs, we adapt the least cost cell, Russell's approximation, and Vogel's approximation methods in the fuzzy context with four indexes to obtain the initial basic feasible solution. Of note, FLC4, FRAM4, and FVAM4 are the adapted methods, respectively. The degeneracy case is handled by the detective method to complete the base of the initial solution. Finally, we will improve this solution using [27] algorithm's second phase to obtain the optimal solution of $\mathrm{FFTP}_{4}$. In the numerical simulation, we will present a comparative study between FLC4, FRAM4, and FVAM4 in various FFTP $_{4}$ examples of different sizes (from $8 \times 16$ to $113 \times 630000$ ). Based on these results, we will choose a suitable algorithm that provides the initial solution that is close to the optimum in a less number of iterations and time.

This paper is organized as follows. Section 2 contains a brief introduction to fuzzy logic, its application and some fuzzy preliminaries. In Section 3, we introduce an economic interpretation of the $\mathrm{FFTP}_{4}$ as well as its associated mathematical formulation. In Section 4, we present the different descriptions of the adapted algorithms. Numerical implementation and a comparative study between FLC4, FRAM4 and FVAM4 are conducted in Section 5. Finally, Section 6 is the conclusion.

\section{Introduction to fuzzy mathematics}

\subsection{Fuzzy logic}

The fuzzy logic is an extension of Boolean logic, it was created in 1965 by L. Zadeh [24] in order to represent mathematically the imprecision, inaccuracies and uncertainties of systems and natural phenomena, by introducing the degree of truth in the verification of a condition; which means a condition can be in state between 0 and 1 (not totally true and not totally 
false but true and false with a degree), like the notion of beauty which is subject to several discussion. Fuzzy logic uses an imprecise but very descriptive language to deal with input data more like a human operator.

\subsection{Fuzzy logic applications}

During the 90s, fuzzy logic becomes a fashion, several researches are published about its applications in various fields (decision making, diagnosis, database, optimization, fuzzy systems control, maintenance of rotating machines, ..., etc.), where no deterministic model exists or is not practically implementable, as well as in situations where the imprecision of the data makes control by classical methods impossible.

\subsection{Preliminaries}

\subsubsection{Fuzzy set}

L. Zadeh defines a fuzzy set as follows: "a fuzzy set is a class with a continuum of membership grades". Thus, a fuzzy set $\tilde{A}$ (fuzzy subset of universe of discourse $U$ ) is characterized by a membership function. It is defined as a set of couples $\left(x, \mu_{\tilde{A}}(x)\right)$, where $x$ is an element of the universe of discourse $U$, and $\mu_{\tilde{A}}$ is the membership function.

\subsubsection{Membership function}

Instead of imposing the membership of an element on "true" or "false " sets, as in the traditional binary logic, fuzzy logic allows degrees of membership. The degree of membership of a fuzzy set is materialized by a number between 0 and 1 . Let $U$ be a set; then, the membership function is defined as $\mu: U \rightarrow[0,1]$. The exact value of the membership function linked to the value of the variable is called the "membership factor". Of note, this membership function is the equivalent of the characteristic function of a classical set.

\subsection{Fuzzy numbers}

A fuzzy number $\tilde{A}$ is a fuzzy subset of the set of real numbers $\mathbb{R}$ with a membership function $\mu_{\tilde{A}}$.

\subsubsection{Trapezoidal fuzzy number}

A fuzzy number $\tilde{A}=(a, b, c, d)$ with $a \leq b \leq c \leq d$ is called a trapezoidal fuzzy number if its membership function is given by:

$$
\mu_{\tilde{A}}(x)=\left\{\begin{array}{cl}
0 & \text { if } x<a \\
\frac{x-a}{b-a} & \text { if } a \leq x \leq b \\
1 & \text { if } b \leq x \leq c \\
\frac{d-x}{d-c} & \text { if } c \leq x \leq d \\
0 & \text { if } d<x
\end{array}\right.
$$

The function $\mu_{\tilde{A}}: \mathbb{R} \rightarrow[0,1]$ is continuous, strictly increasing on $[a, b]$ constant on $[b, c]$ and strictly decreasing on $[c, d]$. It is presented by a trapezoidal shape. 


\subsubsection{Triangular fuzzy number}

Triangular fuzzy number is a particular case of trapezoidal fuzzy number, we get it only if $b=c$ in the representation $(a, b, c, d)$. Its membership function is given by:

$$
\mu_{\tilde{A}}(x)=\left\{\begin{array}{cl}
0 & \text { if } x<a \\
\frac{x-a}{b-a} & \text { if } a \leq x \leq b \\
\frac{d-x}{d-b} & \text { if } b \leq x \leq d \\
0 & \text { if } d<x
\end{array}\right.
$$

The function $\mu_{\tilde{A}}: \mathbb{R} \rightarrow[0,1]$ is continuous, strictly increasing on $[a, b]$ and strictly decreasing on $[c, d]$. It is presented by a triangle shape.

\subsection{Ranking function}

Let $F(\mathbb{R})$ be the set of fuzzy numbers. The ranking function is a defuzification tool of fuzzy numbers to crisp numbers $\Re: F(\mathbb{R}) \rightarrow \mathbb{R}$. It is used to compare fuzzy numbers.

- if $F(\mathbb{R})$ represents a set of trapezoidal fuzzy numbers,

$$
\Re(\tilde{A})=\frac{a+b+c+d}{4}, \quad \tilde{A}=(a, b, c, d),
$$

- if $F(\mathbb{R})$ represents a set of triangular fuzzy numbers,

$$
\Re(\tilde{A})=\frac{a+2 b+d}{4}, \quad \tilde{A}=(a, b, d) .
$$

For two fuzzy numbers $\tilde{A}$ and $\tilde{B}$, we have

$$
\begin{aligned}
\tilde{A} \leq_{\Re} \tilde{B} & \Longleftrightarrow \Re(\tilde{A}) \leq \Re(\tilde{B}), \\
\tilde{A} \geq_{\Re} \tilde{B} & \Longleftrightarrow \Re(\tilde{A}) \geq \Re(\tilde{B}), \\
\tilde{A}=_{\Re} \tilde{B} & \Longleftrightarrow \Re(\tilde{A})=\Re(\tilde{B}) .
\end{aligned}
$$

\subsection{Arithmetic operations}

Let $\tilde{A}=\left(a_{1}, a_{2}, a_{3}, a_{4}\right)$ and $\tilde{B}=\left(b_{1}, b_{2}, b_{3}, b_{4}\right)$ be two fuzzy trapezoidal numbers:

Addition: $\tilde{A} \oplus \tilde{B}=\left(a_{1}, a_{2}, a_{3}, a_{4}\right) \oplus\left(b_{1}, b_{2}, b_{3}, b_{4}\right)=\left(a_{1}+b_{1}, a_{2}+b_{2}, a_{3}+b_{3}, a_{4}+b_{4}\right)$.

Subtraction: $\tilde{A} \ominus \tilde{B}=\left(a_{1}, a_{2}, a_{3}, a_{4}\right) \ominus\left(b_{1}, b_{2}, b_{3}, b_{4}\right)=\left(a_{1}-b_{4}, a_{2}-b_{3}, a_{3}-b_{2}, a_{4}-b_{1}\right)$.

\section{Multiplication:}

- When $\Re(\tilde{A})>0$,

$$
\tilde{A} \otimes \tilde{B}=\left(a_{1} \times \Re(\tilde{B}), a_{2} \times \Re(\tilde{B}), a_{3} \times \Re(\tilde{B}), a_{4} \times \Re(\tilde{B})\right) .
$$

- When $\Re(\tilde{A})<0$,

$$
\tilde{A} \otimes \tilde{B}=\left(a_{4} \times \Re(\tilde{B}), a_{3} \times \Re(\tilde{B}), a_{2} \times \Re(\tilde{B}), a_{1} \times \Re(\tilde{B})\right) .
$$




\subsection{Properties}

- The addition of fuzzy numbers is associative and commutative :

$$
\tilde{A} \oplus \tilde{B}=\tilde{B} \oplus \tilde{A} \text { and } \tilde{A} \oplus(\tilde{B} \oplus \tilde{C})=(\tilde{A} \oplus \tilde{B}) \oplus \tilde{C} \quad \forall \tilde{A}, \tilde{B}, \tilde{C} \in F(\mathbb{R}) .
$$

- No element $\tilde{A} \in F(\mathbb{R})$ has an opposite in $F(\mathbb{R})$.

- The multiplication of a fuzzy number $\tilde{A}=\left(a_{1}, a_{2}, a_{3}, a_{4}\right)$ by a real number $\alpha>0$ is defined by: $\alpha \tilde{A}=\left(\alpha a_{1}, \alpha a_{2}, \alpha a_{3}, \alpha a_{4}\right)$.

- The multiplication of a fuzzy number $\tilde{A}=\left(a_{1}, a_{2}, a_{3}, a_{4}\right)$ by a real number $\alpha<0$ is defined by: $\alpha \tilde{A}=\left(\alpha a_{4}, \alpha a_{3}, \alpha a_{2}, \alpha a_{1}\right)$.

- $(\alpha+\beta) \tilde{A}=\alpha \tilde{A} \oplus \beta \tilde{A} \quad \forall \alpha, \beta \in \mathbb{R}$ with $\alpha \beta \geq 0$ and $\forall \tilde{A} \in F(\mathbb{R})$.

- $\alpha(\tilde{A} \oplus \tilde{B})=\alpha \tilde{A} \oplus \alpha \tilde{B} \quad \forall \alpha \in \mathbb{R}$ and $\forall \tilde{A}, \tilde{B} \in F(\mathbb{R})$.

\section{Four index fuzzy transportation problem}

Let:

- $A_{1}, \ldots, A_{m}, m$ be the origins of availabilities $\tilde{\alpha}_{1}, \ldots, \tilde{\alpha}_{m}$, respectively.

- $B_{1}, \ldots, B_{n}, n$ be the destinations of demands $\tilde{\beta}_{1}, \ldots, \tilde{\beta}_{n}$, respectively.

- $S_{1}, \ldots, S_{p}, p$ be the means of transportation chosen depending on reserved charges $\tilde{\gamma}_{1}, \ldots, \tilde{\gamma}_{p}$ respectively.

- $H_{1}, \ldots, H_{q}, q$ be the qualities of goods obtained in even units of quantities $\tilde{\delta}_{1}, \ldots, \tilde{\delta}_{q}$ respectively.

- $\tilde{x}_{i j k l}(i=1, \ldots, m, j=1, \ldots, n, k=1, \ldots, p, l=1, \ldots, q)$, be the quantity of product $H_{l}$ transported from the origin $A_{i}$ towards the destination $B_{j}$ using the means of transportation $S_{k}$.

- $\tilde{c}_{i j k l}(i=1, \ldots, m, j=1, \ldots, n, k=1, \ldots, p, l=1, \ldots, q)$, be the unit cost of transport of product $\tilde{x}_{i j k l}$.

Mathematically, a four index fuzzy transportation problem can be stated as follows:

$$
\left\{\begin{array}{c}
\text { Minimize } \tilde{Z}=\Re \sum_{i=1}^{m} \sum_{j=1}^{n} \sum_{k=1}^{p} \sum_{l=1}^{q} \tilde{c}_{i j k l} \otimes \tilde{x}_{i j k l} \\
\text { subject to the constraints: } \\
\sum_{j=1}^{n} \sum_{k=1}^{p} \sum_{l=1}^{q} \tilde{x}_{i j k l}={ }_{\Re} \tilde{\alpha}_{i} \text { for all } i=1, \ldots, m \\
\sum_{i=1}^{m} \sum_{k=1}^{p} \sum_{l=1}^{q} \tilde{x}_{i j k l}=\Re \tilde{\beta}_{j} \text { for all } j=1, \ldots, n \\
\sum_{i=1}^{m} \sum_{j=1}^{n} \sum_{l=1}^{q} \tilde{x}_{i j k l}=\Re \tilde{\gamma}_{k} \text { for all } k=1, \ldots, p \\
\sum_{i=1}^{m} \sum_{j=1}^{n} \sum_{k=1}^{p} \tilde{x}_{i j k l}=\Re \tilde{\delta}_{l} \text { for all } l=1, \ldots, q \\
\tilde{x}_{i j k l} \geq_{\Re} 0, \text { for all } i=1, \ldots, m, j=1, \ldots, n, k=1, \ldots, p, l=1, \ldots, q,
\end{array}\right.
$$

where for all $(i, j, k, l)$, we have $\tilde{\alpha}_{i}>_{\Re} 0, \tilde{\beta}_{j}>_{\Re} 0, \tilde{\gamma}_{k}>_{\Re} 0, \tilde{\delta}_{l}>_{\Re} 0$, and $\tilde{c}_{i j k l} \geq_{\Re} 0$. 
We can also write the four index fully fuzzy transportation problem as the following linear program:

$$
\left\{\begin{array}{c}
\min \tilde{Z}={ }_{\Re} \tilde{c}^{T} \otimes \tilde{x} \\
\text { s.c } \\
A \tilde{x}=\Re \tilde{b} \\
\Re(\tilde{x}) \geq 0,
\end{array}\right.
$$

where:

- $\tilde{x}=\left(\tilde{x}_{1111}, \ldots, \tilde{x}_{m n p q}\right)^{T} \in F(\mathbb{R})^{N}$.

- $\tilde{c}=\left(\tilde{c}_{1111}, \ldots, \tilde{c}_{m n p q}\right)^{T} \in F(\mathbb{R})^{N}$.

- $\tilde{b}=\left(\tilde{\alpha}_{1}, \ldots, \tilde{\alpha}_{m}, \tilde{\beta}_{1}, \ldots, \tilde{\beta}_{n}, \tilde{\gamma}_{1}, \ldots, \tilde{\gamma}_{p}, \tilde{\delta}_{1}, \ldots, \tilde{\delta}_{q}\right) \in F(\mathbb{R})^{M}$.

- $A$ is the $M \times N$ matrix with coefficients in $\mathbb{R}$.

- $M=m+n+p+q$ and $N=m n p q$ * $^{*}$

Let $E=\{(i, j, k, l) ; i=1: m, j=1: n, k=1: p$ and $l=1, q\}$ associate for each $(i, j, k, l) \in E$ a vector $P_{i j k l} \in \mathbb{R}^{M}$. Only four components of the $P_{i j k l}$ vector are non-zero; they are located in the lines $i, m+j, m+n+k$, and $m+n+p+l$ and have 1 as a common value. We define the matrix $A$ as the matrix of vectors $P_{i j k l}$. Of note, the matrix $A$ is of $\operatorname{rank} M-3$.

The problem is to determine $\tilde{x}_{i j k l}$ so that the total cost of transport is minimal.

By generalizing the feasible condition in [27], we obtain the following theorem:

Theorem 1. The four index fully fuzzy transportation problem has a feasible solution if and only if

$$
\sum_{i=1}^{m} \tilde{\alpha}_{i}=\Re \sum_{j=1}^{n} \tilde{\beta}_{j}=\Re \sum_{k=1}^{p} \tilde{\gamma}_{k}=\Re \sum_{l=1}^{q} \tilde{\delta}_{l} .
$$

\section{Resolution}

To obtain the fuzzy optimal solution for a four index fully fuzzy transportation problem, we go through two phases:

1. Determining an initial basic feasible solution.

2. Improving a basic feasible solution.

\subsection{Phase 1}

There are three popular methods used to determine an initial feasible solution to the classical transportation problem. They are the least cost, Russell's approximation, and Vogel's approximation methods. We are going to adapt these methods to the four index transportation problem in a fuzzy context. We denote, FLC4, FRAM4, and FVAM4 the adapted algorithms, respectively.

Now, we introduce the description of each method.

Let $E_{b}$ be the set of interesting cells. At the beginning of each algorithm, $E_{b}=\emptyset$. 


\subsubsection{FLC4 algorithm}

The FLC4 algorithm is based on the least cost cell method; the principle of this method is to determine in each step the quantity transported with the minimum cost.

\section{Step 1:}

1. For each unsaturated quadruplet $(i, j, k, l)$, choose $(\bar{i}, \bar{j}, \bar{k}, \bar{l})$, where $\tilde{c}_{\bar{i} \bar{j} \bar{k} \bar{l}}=\min \tilde{c}_{i j k l}$.

2. Take $\tilde{x}_{\bar{i} \bar{j} \bar{k} \bar{l}}=\min \left(\tilde{\alpha}_{\bar{i}}, \tilde{\beta}_{\bar{j}}, \tilde{\gamma}_{\bar{k}}, \tilde{\delta}_{\bar{l}}\right)$ and add $(\bar{i}, \bar{j}, \bar{k}, \bar{l})$ to $E_{b}$.

3. Update $\tilde{\alpha}_{\bar{i}}, \tilde{\beta}_{\bar{j}}, \tilde{\gamma}_{\bar{k}}$ and $\tilde{\delta}_{\bar{l}}$ as follows:

(a) $\tilde{\alpha}_{\bar{i}}=\tilde{\alpha}_{\bar{i}} \ominus \tilde{x}_{\bar{i} \bar{j} \bar{k} \bar{l}}$

if $\tilde{x}_{\bar{i} \bar{j} \bar{k} \bar{l}}=\tilde{\alpha}_{\bar{i}}$ then let $\tilde{x}_{\bar{i} j k l}$ be equal to fuzzy zero and saturate $\tilde{c}_{\bar{i} j k l}, \forall(j, k, l) \neq$ $(\bar{j}, \bar{k}, \bar{l})$.

(b) $\tilde{\beta}_{\bar{j}}=\tilde{\beta}_{\bar{j}} \ominus \tilde{x}_{\bar{i} \bar{j} \bar{k} \bar{l}}$

if $\tilde{x}_{\bar{i} \bar{j} \bar{k} \bar{l}}=\tilde{\beta}_{\bar{j}}$ then let $\tilde{x}_{i \bar{j} k l}$ be equal to fuzzy zero and saturate $\tilde{c}_{i \bar{j} k l}, \forall(i, k, l) \neq(\bar{i}, \bar{k}, \bar{l})$.

(c) $\tilde{\gamma}_{\bar{k}}=\tilde{\gamma}_{\bar{k}} \ominus \tilde{x}_{\bar{i} \bar{j} \bar{k} \bar{l}}$

if $\tilde{x}_{\bar{i} \bar{j} \bar{k} \bar{l}}=\tilde{\gamma}_{\bar{k}}$ then let $\tilde{x}_{i j \bar{k} l}$ be equal to fuzzy zero and saturate $\tilde{c}_{i j \bar{k} l}, \forall(i, j, l) \neq(\bar{i}, \bar{j}, \bar{l})$.

(d) $\tilde{\delta}_{\bar{l}}=\tilde{\delta}_{\bar{l}} \ominus \tilde{x}_{\bar{i} \bar{k} \bar{k} \bar{l}}$

if $\tilde{x}_{\bar{i} \bar{j} \bar{k} \bar{l}}=\tilde{\delta}_{\bar{l}}$ then let $\tilde{x}_{i j k \bar{l}}$ be equal to fuzzy zero and saturate $\tilde{c}_{i j k \bar{l}}, \forall(i, j, k) \neq(\bar{i}, \bar{j}, \bar{k})$.

\section{Step 2:}

- Repeat from 1 to 3 until all $\tilde{x}_{i j k l}$ variables are determined.

\subsubsection{FRAM4 algorithm}

The FRAM4 algorithm is based on Russell's approximation method [20]. The idea of this method is to determine in each step the minimum reduced cost matrix. Then, the transported quantity is chosen as the minimum of this matrix.

\section{Step 1:}

1. For each $i$ unsaturated, determine $\tilde{c}^{i}=\max _{j k l} \tilde{c}_{i j k l}$.

2. For each $j$ unsaturated, determine $\tilde{c}^{j}=\max _{i k l} \tilde{c}_{i j k l}$.

3. For each $k$ unsaturated, determine $\tilde{c}^{k}=\max _{i j l} \tilde{c}_{i j k l}$.

4. For each $l$ unsaturated, determine $\tilde{c}^{l}=\max _{i j k} \tilde{c}_{i j k l}$.

5. Calculate the reduced cost matrix $c_{i j k l}^{*}=\tilde{c}_{i j k l} \ominus\left(\tilde{c}^{i} \oplus \tilde{c}^{j} \oplus \tilde{c}^{k} \oplus \tilde{c}^{l}\right)$.

6. Choose the cell $(\bar{i}, \bar{j}, \bar{k}, \bar{l})$ with the smallest fuzzy reduced cost $c_{i j k l}^{*}$; if there is more than one, choose the one with the smallest fuzzy cost $\tilde{c}_{i j k l}$; if there is equality again, choose the one whose $\min \left(\tilde{\alpha}_{i}, \tilde{\beta}_{j}, \tilde{\gamma}_{k}, \tilde{\delta}_{l}\right)$ is the largest.

7. Take $\tilde{x}_{\bar{i} \bar{j} \bar{k} \bar{l}}=\min \left(\tilde{\alpha}_{\bar{i}}, \tilde{\beta}_{\bar{j}}, \tilde{\gamma}_{\bar{k}}, \tilde{\delta}_{\bar{l}}\right)$ and add $(\bar{i}, \bar{j}, \bar{k}, \bar{l})$ to $E_{b}$.

8. Update $\tilde{\alpha}_{\bar{i}}, \tilde{\beta}_{\bar{j}}, \tilde{\gamma}_{\bar{k}}$ and $\tilde{\delta}_{\bar{l}}$ as in step 1 (3) in the FLC4 algorithm.

\section{Step 2:}

- Repeat from 1 to 8 until all $\tilde{x}_{i j k l}$ variables are determined. 


\subsubsection{FVAM4 algorithm}

The FVAM4 algorithm is based on Vogel's approximation method. This method relies on the minimization of a system of penalties. A penalty of dimension $i, j, k$ or $l$ is the fuzzy difference between the smallest and next smallest fuzzy cost.

\section{Step 1:}

1. For each $i$ unsaturated, determine the penalty $p_{i}^{1}=\min _{2}^{i} \ominus \min _{1}^{i}$.

2. For each $j$ unsaturated, determine the penalty $p_{j}^{2}=\min _{2}^{j} \ominus \min _{1}^{j}$.

3. For each $k$ unsaturated, determine the penalty $p_{k}^{3}=\min _{2}^{k} \ominus \min _{1}^{k}$.

4. For each $l$ unsaturated, determine the penalty $p_{l}^{4}=\min _{2}^{l} \ominus \min _{1}^{l}$.

5. Identify the dimension corresponding to the highest penalty.

6. In the selected dimension found in previous step, identify the cell $(\bar{i}, \bar{j}, \bar{k}, \bar{l})$ with the smallest fuzzy cost.

7. Take $\tilde{x}_{\bar{i} \bar{j} \bar{k} \bar{l}}=\min \left(\tilde{\alpha}_{\bar{i}}, \tilde{\beta}_{\bar{j}}, \tilde{\gamma}_{\bar{k}}, \tilde{\delta}_{\bar{l}}\right)$ and add $(\bar{i}, \bar{j}, \bar{k}, \bar{l})$ to $E_{b}$.

8. Update $\tilde{\alpha}_{\bar{i}}, \tilde{\beta}_{\bar{j}}, \tilde{\gamma}_{\bar{k}}$ and $\tilde{\delta}_{\bar{l}}$ as in step 1 (3) in the FLC4 algorithm.

\section{Step 2:}

- Repeat from 1 to 8 until all $\tilde{x}_{i j k l}$ variables are determined.

\subsection{Treatment of degeneracy}

At the end of phase 1, we obtain the initial feasible solution, which can be degenerate or not degenerate.

Let $I=\left\{(i, j, k, l) ; \Re\left(\tilde{x}_{i j k l}\right)>0\right\}$, and $A_{x}$ is the matrix of vectors $P_{i j k l} \forall(i, j, k, l) \in E_{b}$.

\subsubsection{Test of degeneracy}

- if $\operatorname{rank}\left(A_{x}\right)=\operatorname{rank}(A)$ then

the obtained solution is not degenerate.

- elseif $\operatorname{rank}\left(A_{x}\right)<\operatorname{rank}(A)$ then

the obtained solution is degenerate.

\subsubsection{Treatment of degeneracy}

Let $N_{b}$ be the number of elements of $E_{b}$ that are in the solution, let $E_{h}$ be the complement of $E_{b}$ in the ensemble $E$ (i.e., $E_{h}=E_{b}^{c}$ ), and let $s=\operatorname{rank}(A)-N_{b}$.

- if $N_{b}=\operatorname{rank}(A)$ (i.e., $s=0$ ), then the base is complete and $I^{(0)}=E_{b}$

- if $N_{b}<\operatorname{rank}(A)$ (i.e., $s>0$ ), then

the solution is degenerate; we define the ensemble $E_{s}$ with $s$ elements from $E_{h}$ that are chosen randomly until $E_{b} \cup E_{s}$ is linearly independent and take $I^{(0)}=E_{b} \cup E_{s}$. It is a modification of the method in [28]. 


\subsection{Phase 2}

To test the optimality or to improve the basic feasible solution, we will adapt the phase 2 of $A L_{P T 4 C}[27]$.

1. Initialization:

Let $I^{(r)}$ be the set of interesting quadruplet $(i, j, k, l)$ in iteration $r$. First, take $\mathbf{r}=\mathbf{0} ; I^{(0)}$ was previously defined.

2. For all $(i, j, k, l) \in I^{(r)}$, solve the linear system

$$
A_{x} Y=\Re \tilde{c}_{i j k l} \quad \text { where } \quad Y=\left[\tilde{u}_{i}^{(r)}, \tilde{v}_{j}^{(r)}, \tilde{w}_{k}^{(r)}, \tilde{t}_{l}^{(r)}\right] ; i=1: m, j=1: n, k=1: p, l=1: q .
$$

3. For all $(i, j, k, l) \notin I^{(r)}$ determine

$$
\tilde{\Delta}_{i j k l}^{(r)}=\tilde{c}_{i j k l} \ominus\left(\tilde{u}_{i}^{(r)} \oplus \tilde{v}_{j}^{(r)} \oplus \tilde{w}_{k}^{(r)} \oplus \tilde{t}_{l}^{(r)}\right)
$$

4. - If $\forall(i, j, k, l) \notin I^{(r)}$, we have $\Re\left(\tilde{\Delta}_{i j k l}^{(r)}\right) \geq 0$ then the solution $\tilde{x}^{(r)}$ is optimal. Stop.

- Else use

$$
\tilde{\Delta}_{i_{0} j_{0} k_{0} l_{0}}^{(r)}=\min \left\{\tilde{\Delta}_{i j k l}^{(r)} ; \Re\left(\tilde{\Delta}_{i j k l}^{(r)}\right)<0\right\} .
$$

(a) For all $(i, j, k, l) \in I^{(r)}$, determine a cycle $\mu^{(r)}$ by solving the system

$$
\sum \lambda_{i j k l}^{(r)} A_{x}=-P_{i_{0} j_{0} k_{0} l_{0}}
$$

(b) Determine $\theta^{(r)}$ where $\theta^{(r)}=\min \left(\frac{\tilde{x}_{i j k l}^{(r)}}{-\lambda_{i j k l}^{(r)}}\right)=\theta_{i_{s} j_{s} k_{s} l_{s}}^{(r)}$ with $\lambda_{i j k l}^{(r)}<0$

(c) Determine a new set of basic solution $\tilde{x}^{(r+1)}$ and basic cells as follows:

$$
\begin{gathered}
\tilde{x}^{(r+1)}=\left\{\tilde{x}_{i j k l}^{(r)} /(i, j, k, l) \notin \mu^{(r)}\right\} \cup\left\{\tilde{x}_{i j k l}^{(r)} \oplus \lambda_{i j k l}^{(r)} \theta^{(r)} /(i, j, k, l) \in \mu^{(r)}\right\} . \\
I^{(r+1)}=\left\{I^{(r)} \cup\left\{\left(i_{0}, j_{0}, k_{0}, l_{0}\right)\right\}\right\} \backslash\left\{\left(i_{s}, j_{s}, k_{s}, l_{s}\right\}\right.
\end{gathered}
$$

(d) Repeat steps 1)...4).

\section{Numerical implementation and comparative study}

In this section, we will provide a numerical experiment to test the effectiveness of our adapted algorithms.

Of note, for simplicity, the ranking function of a fuzzy number $(a, b, c)$ is $\Re$ instead of $\Re(a, b, c)$.

\subsection{Example}

In the following example, $\tilde{x}_{B}^{(r)}$ and $\tilde{x}_{H}^{(r)}$ are the set of basic variables and non-basic variables at iteration $r$, respectively.

The transportation problem is in the form FFTP $_{4}$ with $(m=n=p=q=2)$, whose quantities $\tilde{\alpha}_{i}, \tilde{\beta}_{j}, \tilde{\gamma}_{k}, \tilde{\delta}_{l}$, and $\tilde{c}_{i j k l}$ are given by the following tables. 


\begin{tabular}{|c|c|c|c|c|c|c|c|}
\hline$\tilde{c}_{1111}$ & $\tilde{c}_{1112}$ & $\tilde{c}_{1121}$ & $\tilde{c}_{1122}$ & $\tilde{c}_{1211}$ & $\tilde{c}_{1212}$ & $\tilde{c}_{1221}$ & $\tilde{c}_{1222}$ \\
\hline$(4,5,6)$ & $(0,2,7)$ & $(1,3,5)$ & $(5,6,9)$ & $(3,5,6)$ & $(4,6,9)$ & $(6,7,9)$ & $(2,4,5)$ \\
$\Re=5$ & $\Re=2.75$ & $\Re=3$ & $\Re=6.5$ & $\Re=4.75$ & $\Re=6.25$ & $\Re=7.25$ & $\Re=3.75$ \\
\hline$\tilde{c}_{2111}$ & $\tilde{c}_{2112}$ & $\tilde{c}_{2121}$ & $\tilde{c}_{2122}$ & $\tilde{c}_{2211}$ & $\tilde{c}_{2212}$ & $\tilde{c}_{2221}$ & $\tilde{c}_{2222}$ \\
\hline$(5,6,8)$ & $(6,8,10)$ & $(2,3,7)$ & $(6,8,12)$ & $(7,9,11)$ & $(3,9,7)$ & $(3,4,5)$ & $(4,6,10)$ \\
$\Re=6.25$ & $\Re=8$ & $\Re=3.75$ & $\Re=8.5$ & $\Re=9$ & $\Re=7$ & $\Re=4$ & $\Re=6.5$ \\
\hline
\end{tabular}

Table 1: Matrix of costs

\begin{tabular}{|c|c|c|c|c|c|c|c|}
\hline$\tilde{\alpha}_{1}$ & $\tilde{\alpha}_{2}$ & $\tilde{\beta}_{1}$ & $\tilde{\beta}_{2}$ & $\tilde{\gamma}_{1}$ & $\tilde{\gamma}_{2}$ & $\tilde{\delta}_{1}$ & $\tilde{\delta}_{2}$ \\
\hline$(3,7,7)$ & $(1,2,7)$ & $(3,4,8)$ & $(1,5,6)$ & $(2,2,3)$ & $(2,7,11)$ & $(0,4,6)$ & $(4,5,8)$ \\
$\Re=6$ & $\Re=3$ & $\Re=4.75$ & $\Re=4.25$ & $\Re=2.25$ & $\Re=6.75$ & $\Re=3.5$ & $\Re=5.5$ \\
\hline
\end{tabular}

Table 2: Table of $\tilde{\alpha}_{i}, \tilde{\beta}_{j}, \tilde{\gamma}_{k}$ and $\tilde{\delta}_{l}$ quantities

This FFTP $_{4}$ provides a feasible solution because:

$$
\sum_{i=1}^{2} \tilde{\alpha}_{i}=\Re \sum_{j=1}^{2} \tilde{\beta}_{j}=\Re \sum_{l=1}^{2} \gamma_{k}=\Re \sum_{l=1}^{2} \tilde{\delta}_{l}=(4,9,14)
$$

The size of this problem is $(2,2,2,2)$; thus, here, $M=8$ and $N=16$.

\subsubsection{Application of the FLC4 algorithm}

\section{Step 1}

- Take $E_{b}=\emptyset$,

- $\min \tilde{c}_{i j k j l}=\tilde{c}_{1112}$

- Determine $\tilde{x}_{1112}$ :

$$
\begin{aligned}
\tilde{x}_{1112} & =\min \left(\tilde{\alpha}_{1}, \tilde{\beta}_{1}, \tilde{\gamma}_{1}, \tilde{\delta}_{2}\right) \\
& =\min \left((3,7,7)_{\Re=6},(3,4,8)_{\Re=4.75},(2,2,3)_{\Re=2.25},(4,5,8)_{\Re=5.5}\right) \\
& =(2,2,3)_{\Re=2.25}
\end{aligned}
$$

- Add $(1,1,1,2)$ to $E_{b}$,

- Update $\tilde{\alpha}_{1}, \tilde{\beta}_{1}, \tilde{\gamma}_{1}$ and $\tilde{\delta}_{2}$ as:

$$
\begin{gathered}
\tilde{\alpha}_{1}=(0,5,5), \\
\tilde{\beta}_{1}=(0,2,6), \\
\tilde{\gamma}_{1}=(-1,0,1), \\
\tilde{\delta}_{2}=(1,3,6) .
\end{gathered}
$$

- For all $(i, j, l) \neq(1,1,2)$, let $\tilde{x}_{i j 1 l}$ be equal to fuzzy zero and saturate $\tilde{c}_{i j 1 l}$.

Then, repeat until all $\tilde{x}_{i j k l}$ variables are determined.

The initial basic feasible solution given by FLC4 is: $\tilde{x}^{(0)}=\tilde{x}_{B}^{(0)} \cup \tilde{x}_{H}^{(0)}$ where

$$
\tilde{x}_{B}^{(0)}=\left\{\tilde{x}_{1112}^{(0)}=(2,2,3), \tilde{x}_{1121}^{(0)}=(0,2,6), \tilde{x}_{1222}^{(0)}=(-6,3,5), \tilde{x}_{2221}^{(0)}=(-6,2,6), \tilde{x}_{2222}=(-4,0,12)\right\}
$$


The value of the objective associated with $\tilde{x}^{(0)}$ is

$$
\tilde{Z}^{(0)}=\Re \sum_{i=1}^{2} \sum_{j=1}^{2} \sum_{k=1}^{2} \sum_{l=1}^{2} \tilde{c}_{i j k l} \otimes \tilde{x}_{i j k l}^{(0)}=(16,33,59.5) .
$$

\section{Test of degeneracy}

The number of elements of $\tilde{x}_{B}^{(0)}$ is equal to $5=M-3$; thus, the solution is not degenerate.

\section{Phase 2}

The test of optimality in phase 2 shows that this solution is not optimal. Thus, we can improve it. $\tilde{x}^{(1)}=\tilde{x}_{B}^{(1)} \cup \tilde{x}_{H}^{(1)}$ where

$\tilde{x}_{B}^{(1)}=\left\{\tilde{x}_{1112}^{(1)}=(2,2,3), \tilde{x}_{1121}^{(1)}=(-12,2,10), \tilde{x}_{1222}^{(1)}=(-10,3,17), \tilde{x}_{2121}^{(1)}=(-4,0,12), \tilde{x}_{2221}^{(1)}=(-6,2,6)\right\}$.

The test of optimality shows that $\tilde{x}^{(1)}$ is optimal. The value of the objective associated with $\tilde{x}^{(1)}$ is:

$$
\tilde{Z}^{(1)}={ }_{\Re} \sum_{i=1}^{2} \sum_{j=1}^{2} \sum_{k=1}^{2} \sum_{l=1}^{2} \tilde{c}_{i j k l} \otimes \tilde{x}_{i j k l}^{(1)}=(14,29,53.5) .
$$

\subsubsection{Application of the FRAM4 algorithm}

- Take $E_{b}=\emptyset$,

- Calculate the reduced costs:

\begin{tabular}{|c|c|c|c|c|c|c|c|}
\hline$\tilde{c}^{(i=1)}$ & $\tilde{c}^{(i=2)}$ & $\tilde{c}^{(j=1)}$ & $\tilde{c}^{(j=2)}$ & $\tilde{c}^{(k=1)}$ & $\tilde{c}^{(k=2)}$ & $\tilde{c}^{(l=1)}$ & $\tilde{c}^{(l=2)}$ \\
\hline$(6,7,9)$ & $(7,9,11)$ & $(6,8,12)$ & $(7,9,11)$ & $(7,9,11)$ & $(6,8,12)$ & $(7,9,11)$ & $(6,8,12)$ \\
\hline
\end{tabular}

Table 3: Reduced costs in the first iteration

- Calculate the reduced costs matrix:

\begin{tabular}{|c|c|c|c|}
\hline$c_{1111}^{*}$ & $c_{1112}^{*}$ & $c_{1121}^{*}$ & $c_{1122}^{*}$ \\
\hline$(-29,-28,-20)$ & $(-44,-30,-18)$ & $(-43,-29,-20)$ & $(-40,-25,-15)$ \\
\hline$c_{1211}^{*}$ & $c_{1212}^{*}$ & $c_{1221}^{*}$ & $c_{1222}^{*}$ \\
\hline$(-39,-29,-21)$ & $(-39,-27,-17)$ & $(-37,-26,-17)$ & $(-42,-28,-20)$ \\
\hline$c_{2111}^{*}$ & $c_{2112}^{*}$ & $c_{2121}^{*}$ & $c_{2122}^{*}$ \\
\hline$(-40,-29,-19)$ & $(-40,-26,-16)$ & $(-44,-31,-19)$ & $(-41,-25,-13)$ \\
\hline$c_{2211}^{*}$ & $c_{2212}^{*}$ & $c_{2221}^{*}$ & $c_{2222}^{*}$ \\
\hline$(-37,-27,-17)$ & $(-42,-26,-20)$ & $(-42,-31,-22)$ & $(-44,-28,-16)$ \\
\hline
\end{tabular}

Table 4: Reduced costs matrix in the first iteration

- $\min _{i, j, k, l} c_{i j k l}^{*}=c_{2221}^{*}$,

- Determine $\tilde{x}_{2221}$ :

$$
\begin{aligned}
\tilde{x}_{2221} & =\min \left(\tilde{\alpha}_{2}, \tilde{\beta}_{2}, \tilde{\gamma}_{2}, \tilde{\delta}_{1}\right) \\
& =\min \left((1,2,7)_{\Re=3},(1,5,6)_{\Re=4.25},(2,7,11)_{\Re=6.75},(0,4,6)_{\Re=3.5}\right) \\
& =(1,2,7)_{\Re=3} .
\end{aligned}
$$


- Add $(2,2,2,1)$ to $E_{b}$,

- Update $\tilde{\alpha}_{2}, \tilde{\beta}_{2}, \tilde{\gamma}_{2}$ and $\tilde{\delta}_{1}$ as:

$$
\begin{gathered}
\tilde{\alpha}_{2}=(-6,0,6), \\
\tilde{\beta}_{2}=(-6,3,5), \\
\tilde{\gamma}_{2}=(-5,5,10), \\
\tilde{\delta}_{1}=(-7,2,5) .
\end{gathered}
$$

- For all $(i, j, l) \neq(2,2,1)$, let $\tilde{x}_{2 j k l}$ be equal to fuzzy zero and saturate $\tilde{c}_{2 j k l}$.

Then, repeat until all $\tilde{x}_{i j k l}$ variables are determined.

The initial basic feasible solution given by the FRAM4 method is $\tilde{x}^{(0)}=\tilde{x}_{B}^{(0)} \cup \tilde{x}_{H}^{(0)}$ where

$\tilde{x}_{B}^{(0)}=\left\{\tilde{x}_{2221}^{(0)}=(1,2,7), \tilde{x}_{1121}^{(0)}=(-7,2,5), \tilde{x}_{1112}^{(0)}=(2,2,3), \tilde{x}_{1222}^{(0)}=(-6,3,5), \tilde{x}_{1122}^{(0)}=(-10,0,18)\right\}$.

The value of the objective associated with $\tilde{x}^{(0)}$ is

$$
\tilde{Z}^{(0)}=\Re \sum_{i=1}^{2} \sum_{j=1}^{2} \sum_{k=1}^{2} \sum_{l=1}^{2} \tilde{c}_{i j k l} \otimes \tilde{x}_{i j k l}^{(0)}=(14,29,53.5) .
$$

Test of degeneracy

The number of elements of $\tilde{x}_{B}^{(0)}$ is equal to $5=M-3$; thus, the solution is not degenerate.

Phase 2

The test of optimality in phase 2 shows that the initial solution obtained from the FRAM4 algorithm is optimal and does not need improvement.

\subsubsection{Application of the FVAM4 algorithm}

\section{Step 1}

- Take $E_{b}=\emptyset$,

- Calculate the penalties:

\begin{tabular}{|c|c|c|c|c|c|c|c|}
\hline$p_{1}^{1}$ & $p_{2}^{1}$ & $p_{1}^{2}$ & $p_{2}^{2}$ & $p_{1}^{3}$ & $p_{2}^{3}$ & $p_{1}^{4}$ & $p_{2}^{4}$ \\
\hline$(-6,1,5)$ & $(-4,1,3)$ & $(-6,1,5)$ & $(-2,0,3)$ & $(-\mathbf{4 , 3 , 6})$ & $(-3,1,4)$ & $(-3,0,6)$ & $(-5,2,5)$ \\
\hline
\end{tabular}

Table 5: Penalties in the first iteration

- $\max \left(p_{i}^{1}, p_{j}^{2}, p_{k}^{3}, p_{l}^{4}\right)=p_{1}^{3}$,

- $\min \tilde{c}_{i j 1 l}=\tilde{c}_{1112}$,

- Determine $\tilde{x}_{1112}$ :

$$
\begin{aligned}
\tilde{x}_{1112} & =\min \left(\tilde{\alpha}_{1}, \tilde{\beta}_{1}, \tilde{\gamma}_{1}, \tilde{\delta}_{2}\right) \\
& =\min \left((3,7,7)_{\Re=6},(3,4,8)_{\Re=4.75},(2,2,3)_{\Re=2.25},(4,5,8)_{\Re=5.5}\right) \\
& =(2,2,3)_{\Re=2.25} .
\end{aligned}
$$

- Add $(1,1,1,2)$ to $E_{b}$, 
- Update $\tilde{\alpha}_{1}, \tilde{\beta}_{1}, \tilde{\gamma}_{1}$ and $\tilde{\delta}_{2}$ as:

$$
\begin{gathered}
\tilde{\alpha}_{1}=(0,5,5), \\
\tilde{\beta}_{1}=(0,2,6), \\
\tilde{\gamma}_{1}=(-1,0,1), \\
\tilde{\delta}_{2}=(1,3,6) .
\end{gathered}
$$

- For all $(i, j, l) \neq(1,1,2)$, let $\tilde{x}_{i j l l}$ be equal to fuzzy zero and saturate $\tilde{c}_{i j 1 l}$.

Then repeat until all $\tilde{x}_{i j k l}$ variables are determined.

The initial basic feasible solution given by the FVAM4 method is: $\tilde{x}^{(0)}=\tilde{x}_{B}^{(0)} \cup \tilde{x}_{H}^{(0)}$, where

$\tilde{x}_{B}^{(0)}=\left\{\tilde{x}_{1112}^{(0)}=(2,2,3), \tilde{x}_{1222}^{(0)}=(1,3,6), \tilde{x}_{1121}^{(0)}=(-6,2,4), \tilde{x}_{2221}^{(0)}=(-5,2,5), \tilde{x}_{2121}^{(0)}=(-4,0,12)\right\}$.

The value of the objective associated with $\tilde{x}^{(0)}$ is

$$
\tilde{Z}^{(0)}=\Re \sum_{i=1}^{2} \sum_{j=1}^{2} \sum_{k=1}^{2} \sum_{l=1}^{2} \tilde{c}_{i j k l} \otimes \tilde{x}_{i j k l}^{(0)}=(14,29,53.5)
$$

\section{Test of degeneracy}

The number of elements of $\tilde{x}_{B}^{(0)}$ is equal to $5=M-3$; thus, the solution is not degenerate.

Phase 2

The test of optimality in phase 2 shows that $\tilde{x}^{(0)}$ is optimal and does not need improvement.

\subsection{Comparative study}

The following tables show a comparative study between the three adapted algorithms FLC4, FRAM4, and FVAM4 that are applied to solve the four index fully fuzzy transportation problem. We will treat 13 different problems with different dimensions $M \times N$. For each problem, a set of data and cost matrixes are randomly chosen for the fair comparison of these algorithms.

Discussion

- These tables show that the proposed algorithms can effectively solve $F F T P_{4}$ with a large range of dimensions from $8 \times 16$ to more than $113 \times 630000$.

- The results show the robustness of our method in phase 2 when finding the optimal solution, from the initial one, even in the case of degeneracy.

- The initial basic feasible solution obtained by FLC4 is far from being the optimal one for most $\mathrm{FFTP}_{4}$; its main advantage is that it is quick and easy, and its simulation code is very simple. 


\begin{tabular}{|c|c|c|c|c|c|c|c|c|}
\hline \multicolumn{2}{|c|}{ Dimension $(M \times N)$} & $12 \times 81$ & $16 \times 265$ & $20 \times 625$ & $24 \times 1296$ & $32 \times 4096$ & $40 \times 1000$ & $64 \times 65536$ \\
\hline \multirow{9}{*}{$\frac{\mathrm{N}}{\mathrm{e}}$} & FLC4 & $(-2998.8$ & $(-21125$ & $(-54986$ & $(-75878$ & $(-62829$ & $(-33481$ & $(-77002$ \\
\hline & & 2093.8 & 1653.3 & 1052.3 & 2136.3 & 1166.5 & 348.5 & 1750 \\
\hline & & $7131.8)$ & 24298) & $57223)$ & 79614) & $65238)$ & 34377) & $80003)$ \\
\hline & FRAM4 & $(-1948.8$ & $(-23479$ & $(-70631$ & $(-45375$ & $(-40825$ & $(-61920$ & $(-1.4375 \mathrm{e} 5$ \\
\hline & & 2043.8 & 1622.5 & $93173088)$ & 1895.8 & 1021.3 & 314.75 & 1432 \\
\hline & & $6307)$ & 26517) & & 49255) & 43588) & 63186) & $1.4748 \mathrm{e} 5)$ \\
\hline & FVAM4 & $(-2836.3$ & $(-29170$ & $(-25078$ & $(-43860$ & $(-60253$ & $(-84944$ & $(-1.1649 \mathrm{e} 5$ \\
\hline & & 2103.3 & 1440.5 & 1094.8 & 1865.8 & 1192.3 & $36085863)$ & 1417.3 \\
\hline & & 6971.3) & 32234) & 26875) & $47852)$ & $62500)$ & & $1.199 \mathrm{e} 5)$ \\
\hline \multirow{3}{*}{$\frac{\sqrt[\pi]{2}}{\sqrt{\mathrm{N}}}$} & FLC4 & 2080.1 & 1619.9 & 1085.6 & 2002.3 & 1185.5 & 398.25 & 1625.3 \\
\hline & FRAM4 & 2111.4 & 1570.8 & 1079.8 & 1917.7 & 1201.4 & 474 & 1649.1 \\
\hline & FVAM4 & 2085.4 & 1486.4 & 996.69 & 1931 & 1158 & 409.69 & 1561 \\
\hline \multirow{3}{*}{ 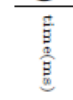 } & FLC4 & 1.5356 & 2.2069 & 4.0085 & 12.481 & 13.846 & 15.576 & 17.398 \\
\hline & FRAM4 & 3.0169 & 6.7252 & 9.512 & 31.602 & 50.462 & 96.994 & 701.71 \\
\hline & FVAM4 & 3.6408 & 6.794 & 7.9264 & 30.963 & 30.922 & 45.034 & 168.96 \\
\hline \multirow{9}{*}{$\underset{*}{*}$} & FLC4 & $(-2818$ & $(-12709$ & $(-27951$ & $(-52205$ & $(-28621$ & $(-18536$ & $(-43864$ \\
\hline & & 2015.8 & 1343.3 & 864.25 & 1455.3 & 847.66 & 284.85 & 682.97 \\
\hline & & 6833.8) & 15325) & 29806) & 54931) & 30335) & 19198) & 45460) \\
\hline & FRAM4 & $(-1900.8$ & $(-10040$ & $(-32677$ & $(-28878$ & $(-19155$ & $(-41649$ & $(-72237$ \\
\hline & & 2015.7 & 1347.3 & 874.75 & 1455.3 & 847.66 & 295.58 & 704.61 \\
\hline & & 5916.5) & 12649) & 34511) & 31605) & 20869) & 42290) & 73789) \\
\hline & FVAM4 & $(-2775.2$ & $(-17652$ & $(-17330$ & $(-29654$ & $(-37737$ & $(-31037$ & $(-79578$ \\
\hline & & 2015.8 & 1345.6 & 868.75 & 1455.3 & 848.3 & 290.22 & 684.73 \\
\hline & & 6791) & 20264) & 19176) & 32381) & 39450) & 31689) & $81170)$ \\
\hline \multirow{3}{*}{ 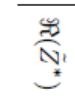 } & FLC4 & 2011.8 & 1325.9 & 895.87 & 1409.3 & 852.24 & 308.01 & 740.36 \\
\hline & FRAM4 & 2011.8 & 1325.9 & 895.87 & 1409.3 & 852.24 & 308.0 & 740.36 \\
\hline & FVAM4 & 2011.8 & 1325.9 & 895.87 & 1409.3 & 852.24 & 308.01 & 740.36 \\
\hline \multirow{3}{*}{ 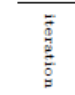 } & FLC4 & 4 & 12 & 14 & 50 & 52 & 69 & 345 \\
\hline & FRAM4 & 3 & 14 & 16 & 48 & 70 & 68 & 418 \\
\hline & FVAM4 & 2 & 8 & 12 & 48 & 57 & 68 & 354 \\
\hline \multirow{3}{*}{$\frac{D}{3}$} & FLC4 & 8.8915 & 50.207 & 145.4 & 1507.8 & 8845.3 & 56340 & $1.0578 \mathrm{e} 5$ \\
\hline & FRAM4 & 7.1789 & 58.461 & 162.94 & 1347.3 & 11605 & 58289 & $1.2892 \mathrm{e} 5$ \\
\hline & FVAM4 & 5.0874 & 34.99 & 125.01 & 1318.6 & 9576.5 & 56062 & $1.0562 \mathrm{e} 5$ \\
\hline \multirow{3}{*}{$\begin{array}{l}\text { Total } \\
\text { time } \\
(\mathrm{ms})\end{array}$} & FLC4 & 10.427 & 52.414 & 149.41 & 1520.3 & 8859.1 & 56356 & $1.058 \mathrm{e} 5$ \\
\hline & FRAM4 & 10.196 & 65.186 & 172.45 & 1378.9 & 11655 & 58386 & $1.2962 \mathrm{e} 5$ \\
\hline & FVAM4 & 8.7282 & 41.784 & 132.94 & 1349.6 & 9607.4 & 56107 & $1.0579 \mathrm{e} 5$ \\
\hline
\end{tabular}

Table 6: FLC4, FRAM4, and FVAM4 in solving FFTP 4

- The initial basic feasible solution obtained by FVAM4 or FRAM4 methods is very close to the optimal one.

- In most cases, FVAM4 requires less time and a smaller number of iterations compared to FRAM4 and FLC4. Consequently, this method is preferable to use to solve the four index fully fuzzy transportation problems with large size. 


\begin{tabular}{|c|c|c|c|c|c|c|c|}
\hline \multicolumn{2}{|c|}{ Dimension $(M \times N)$} & $30 \times 3136$ & $35 \times 5760$ & $37 \times 7200$ & $41 \times 10800$ & $80 \times 16000$ & $\begin{array}{l}113 \\
630000\end{array}$ \\
\hline \multirow{9}{*}{$\stackrel{\mathrm{N}}{\mathrm{\varrho}}$} & FLC4 & $(-1.4195 \mathrm{e} 5$ & $(-30855$ & $(-57572$ & $(-1.2796 \mathrm{e} 5$ & $(-2.6674 \mathrm{e} 5$ & $(-5.4236 \mathrm{e} 5$ \\
\hline & & 1507.8 & 2086.8 & 1157.3 & 1602 & 764.25 & 854 \\
\hline & & $1.4447 \mathrm{e} 5)$ & 34891) & 59759) & $1.3161 \mathrm{e} 5)$ & $2.7078 \mathrm{e} 5)$ & $5.4413 \mathrm{e} 5)$ \\
\hline & FRAM4 & $(-15678$ & $(-26876$ & $(-39550$ & $(-57375$ & $(-6.2504 \mathrm{e} 5$ & $(-1.9974 \mathrm{e} 5$ \\
\hline & & 1711.3 & $192030676)$ & 1127.3 & 1496.3 & 1357 & 920.25 \\
\hline & & 18334) & & 41836) & 60930) & $6.2814 \mathrm{e} 5)$ & $2.0114 \mathrm{e} 5)$ \\
\hline & FVAM4 & $(-30597$ & $(-49972$ & $(-38357$ & $(-47532$ & $(-1.5441 \mathrm{e} 5$ & $(-234661$ \\
\hline & & 1508.5 & 1777.8 & 1067.8 & 1353.8 & 1256 & 885.25 \\
\hline & & 33267) & 52403) & 40487) & $51708)$ & $1.5731 \mathrm{e} 5)$ & 236372) \\
\hline \multirow{3}{*}{$\frac{\pi}{\sqrt{3}}$} & FLC4 & 1384.1 & 2052.3 & 1125.4 & 1714.4 & 1393.4 & 871.31 \\
\hline & FRAM4 & 1519.4 & 1910.1 & 1135.2 & 1636.8 & 1454.9 & 808.81 \\
\hline & FVAM4 & 1421.9 & 1496.6 & 1066.4 & 1721 & 1352.8 & 870.38 \\
\hline \multirow{3}{*}{$\stackrel{\frac{a}{3}}{\stackrel{a}{a}}$} & FLC4 & 13.73 & 6.0158 & 15.905 & 16.345 & 63.173 & 211.78 \\
\hline & FRAM4 & 43.844 & 62.404 & 75.093 & 101.12 & 2148.7 & 15467 \\
\hline & FVAM4 & 30.119 & 24.991 & 39.1 & 72.504 & 382.51 & 2137 \\
\hline \multirow{9}{*}{$\underset{*}{N}$} & FLC4 & $(-76187$ & $(-16392$ & $(-46683$ & $(-72990$ & -83399 & $(-2.375 \mathrm{e} 5$ \\
\hline & & 1080.6 & 853.97 & 800.33 & 933.75 & 555.63 & 417.71 \\
\hline & & $78056)$ & 17984) & 48381) & 75036) & $84528)$ & $2.3825 \mathrm{e} 5)$ \\
\hline & FRAM4 & $(-10714$ & $(-15701$ & $(-25600$ & $(-39633$ & $(-2.8724 \mathrm{e} 5$ & $(-96678$ \\
\hline & & 1108.6 & 853.97 & 795.83 & 940.25 & 530.74 & 412.08 \\
\hline & & 12528) & 17293) & 27307) & 41666) & $2.8842 \mathrm{e} 5)$ & 97440) \\
\hline & FVAM4 & $(-22150$ & $(-29421$ & $(-34851794$ & $(-47532$ & $(-97204$ & $(-1.4737 \mathrm{e} 5$ \\
\hline & & 1137.7 & 871.09 & $36562)$ & 1353.8 & 540.54 & 418.57 \\
\hline & & 23905) & 30979) & & $51708)$ & 98363) & $1.4812 \mathrm{e} 5)$ \\
\hline \multirow{3}{*}{$\underset{*}{\stackrel{*}{*}}$} & FLC4 & 1007.6 & 825.09 & 824.65 & 978.38 & 560 & 396.65 \\
\hline & FRAM4 & 1007.6 & 825.09 & 824.65 & 978.38 & 560 & 396.65 \\
\hline & FVAM4 & 1007.6 & 825.09 & 824.65 & 978.38 & 560 & 396.65 \\
\hline \multirow{3}{*}{ 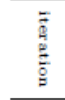 } & FLC4 & 58 & 86 & 83 & 130 & 565 & 1225 \\
\hline & FRAM4 & 68 & 86 & 79 & 123 & 549 & 1095 \\
\hline & FVAM4 & 60 & 83 & 88 & 91 & 512 & 1074 \\
\hline \multirow{3}{*}{ 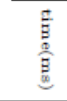 } & FLC4 & 1103.7 & 3452.1 & 3785 & 7341 & $4.3418 \mathrm{e} 5$ & $3.6585 \mathrm{e} 6$ \\
\hline & FRAM4 & 1959.5 & 2813.9 & 3334.3 & 8074.2 & $4.1115 \mathrm{e} 5$ & $3.1405 \mathrm{e} 6$ \\
\hline & FVAM4 & 1245.2 & 4161.7 & 3704.6 & 5824.4 & $3.9497 \mathrm{e} 5$ & $3.01 \mathrm{e} 6$ \\
\hline \multirow{3}{*}{$\begin{array}{l}\text { Total } \\
\text { time } \\
(\mathrm{ms})\end{array}$} & FLC4 & 1117.4 & 3458.1 & 3800.9 & 7357.4 & $4.3424 \mathrm{e} 5$ & $3.6587 \mathrm{e} 6$ \\
\hline & FRAM4 & 2003.4 & 2876.3 & 3409.4 & 8175.3 & $4.133 \mathrm{e} 5$ & $3.156 \mathrm{e} 6$ \\
\hline & FVAM4 & 1275.3 & 4186.7 & 3743.7 & 5896.9 & $3.9535 \mathrm{e} 5$ & $3.0121 \mathrm{e} 6$ \\
\hline
\end{tabular}

Table 7: FLC4, FRAM4, and FVAM4 in solving FFTP 4

\section{Conclusion}

In many real-life situations, data from transportation problems are often uncertain. To deal with such problems, this uncertainty can be modeled using fuzzy mathematics. In this study, we proposed three approaches (i.e., FLC4, FRAM4, and FVAM4) to determine the initial basic feasible solution to the four index fully fuzzy transportation. Then, after treating the degeneracy problem, we initiate the second phase to determine the optimal solution. Of note, the arithmetic operations used are based on the notion of ranking function. We performed numerical experiments to test the efficiency and stability of our algorithms. The obtained results are encouraging and show that the method, in general, provides the initial solution close to the optimum. The algorithms FLC4, FRAM4, FVAM4 are independent of the number of indexes, as shown in the numerical examples. Therefor a comparative table can be used to solve fuzzy multi-index problems. The second phase of the algorithm has shown its robustness and efficiency for determining the optimal solution in a considerably shorter period of time. 


\section{Acknowledgements}

This work was made possible by the Direction Générale de la Recherche Scientifique et du Développement Technologique (DGRSDT), MESRS Algeria, and research project PRFU under code: C00L03UN190120180008.

We would like to express our sincere appreciation and gratitude to the anonymous peer reviewers for their insightful comments, which greatly improved the manuscript. We are also immensely grateful to Mr. Jean-Pierre CROUZEIX, Emeritus Professor at ISIMA/LIMOS, University of Clermont Auvergne, France, for his helpful comments and suggestions on an earlier version of this manuscript, although any errors are our own and should not tarnish the reputation of this respected person.

\section{References}

[1] Baykasoğlu, A., and Subulan, K. (2019). A direct solution approach based on constrained fuzzy arithmetic and metaheuristic for fuzzy transportation problems. Soft Computing, 23(5), 16671698. doi: 10.1007/s00500-017-2890-2

[2] Bellman, R. E. and Zadeh, L. A. (1970). Decision-making fuzzy environment. Management Science, 17(4), 141-164. doi: 10.1287/mnsc.17.4.B141

[3] Chanas, S., Kolodziejczy, W., and Machaj, A. (1984). A fuzzy approach to the transportation problem. Fuzzy Sets and Systems, 13(3), 211-221. doi: 10.1016/0165-0114(84)90057-5

[4] Chanas, S., and Kuchta, D. (1996). A concept of optimal solution of the transportation with Fuzzy cost coefficient. Fuzzy sets and systems, 82(3), 299-305. doi: 10.1016/0165-0114(95)00278-2

[5] Ebrahimnejad, A., Verdegay, J. L. (2018). A new approach for solving fully intuitionistic fuzzy transportation problems. Fuzzy Optimization and Decision Making, 17(4), 447-474. doi: 10.1007/s10700-017-9280-1

[6] Hedid, M., Zitouni, R., Labdai, S. (2020). Adaptive algorithm for solving the four-index fuzzy transportation problem. IEEE 6th International Conference on Optimization and Applications, ICOA 2020, 1-5. doi: 10.1109/icoa49421.2020.9094480

[7] Kaur, A., and Kumar, A. (2011). A new method for solving fuzzy transportation problems using ranking function. Applied Mathematical Modelling, 35(12), 5652-5661. doi: 10.1016/j.apm.2011.05.012

[8] Kumar, R., Edalatpanah, S. A., Jha, S. et al., (2019). A Pythagorean fuzzy approach to the transportation problem. Complex \& Intelligent Systems, 5(2), 255-263. doi: 10.1007/s40747-0190108-1

[9] Kumar, B. R., and Murugesan, S. (2012). On fuzzy transportation problem using triangular fuzzy numbers with modified revisred simplex method. International Journal of Engineering, Science and Technology, 4(1), 285-294.

[10] Kumari, P. (2014). A Comparative study of optimization methods for fuzzy transportation problems. International Journal of Scientific \& Engineering Research, 5, 76-80.

[11] Liu, S.-T., and Kao, C. (2004). Solving fuzzy transportation problems based on extension principle. European Journal of Operational Research, 153(3), 661-674. doi: 10.1016/S0377-2217(02)00731-2

[12] Mahmoodirad, A., Allahviranloo, T., and Niroomand, S. (2019). A new effective solution method for fully intuitionistic fuzzy transportation problem. Soft Computing, 23(12), 4521-4530. doi: $10.1007 / \mathrm{s} 00500-018-3115-\mathrm{z}$

[13] Mishra, A., and Kumar, A. (2020). JMD method for transforming an unbalanced fully intuitionistic fuzzy transportation problem into a balanced fully intuitionistic fuzzy transportation problem. Soft Computing, 24(24), 15639-15654. doi: 10.1007/s00500-020-04889-6

[14] Muruganandam, S., and Srinivasan, R. (2016). A new algorithm for solving fuzzy transportation problems with trapezoidal fuzzy numbers. International Journal of Recent Trends in Engineering E) Research, 2, 428-437.

[15] Muthuperumal, S., Titus, P. and Venkatachalapathy, M. (2020). An algorithmic approach to solve unbalanced triangular fuzzy transportation problems. Soft Computing, 24(24), 18689-18698. doi: $10.1007 / \mathrm{s} 00500-020-05103-3$ 
[16] Nagoor Gani, A., and Razak, K. A. (2006). Two stage fuzzy transportation problem. Journal of Physical Sciences, 10, 63-69.

[17] Pandian, P., and Nagarajan, G. (2010). A new algorithm for solving a fuzzy optimal solution for fuzzy transportation problem. Applied Mathematical Sciences, 4(1-2), 79-90. http://www . m-hikari.com/ams/ams-2010/ams-1-4-2010/pandianAMS1-4-2010.pdf

[18] Pham, T.-H., and Dott, P. (2012). An exact method for solving the four index transportation problem and industrial application. American Journal of Operational Research, 3(2), 28-44. doi: 10.5923/j.ajor.20130302.02

[19] Pratihar, J., Kumar, R., Edalatpanah, S. A., and Dey, A. (2020). Modifed Vogel's approximation method for transportation problem under uncertain environment. Complex $\&$ Intelligent Systems. doi: $10.1007 / \mathrm{s} 40747-020-00153-4$

[20] Russell, E. J. (1969). Extension of Dantzig's algorithm for finding an initial near-optimal basis for the transportation problem. Operations Research, 17(1), 187-191. doi: 10.1287/opre.17.1.187

[21] Senthil Kumar, P. (2016a). PSK method for solving type-1 and type-3 fuzzy transportation problems. International Journal of Fuzzy System Applications, 5(4), 121-146. doi: 10.4018/IJFSA.2016100106

[22] Senthil Kumar, P. (2016b). A simple method for solving type-2 and type-4 fuzzy transportation problems. International Journal of Fuzzy Logic and Intelligent Systems, 16(4), 225-237. doi: 10.5391/IJFIS.2016.16.4.225

[23] Singh, S. K., and Yadav, S. P. (2015). Efficient approach for solving type-1 intuitionistic fuzzy transportation problem. International Journal of System Assurance Engineering and Management, 6(3), 259-267. doi: 10.1007/s13198-014-0274-x

[24] Zadah, L. (1965). Fuzzy sets. Information and control, 8(3), 338-353. doi: 10.1016/S00199958(65)90241-X

[25] Zimmermann, H. J. (1978). Fuzzy programming and linear programming with several objective function. Fuzzy Set and Systems, 1(1), 45-55. doi: 10.1016/0165-0114(78)90031-3

[26] Zitouni, R., and Achache, M. (2017). A numerical comparison between two exact methods for solving a capacitated 4-index transportation problem. Journal of Numerical Analysis and Approximation Theory, 46(2), 181-192. https://ictp.acad.ro/jnaat/journal/article/view/1116

[27] Zitouni, R., and Keraghel, A. (2003). Resolution of a capacitated transportation problem with four subscripts. Kybernetes, 32(9-10), 1450-1463. doi: 10.1108/03684920310493341

[28] Zitouni, R., and Keraghel, A. (2007). A note on the algorithm of resolution of a capacitated transportation problem with four subscripts. Far East Journal of Mathematical Sciences (FJMS), 26(3), 769-778. http://www.pphmj.com/abstract/2672.htm 